\title{
ESTUDIO DE FRUTOS Y SEMILLAS DE IPOMOEA TEOTITLANICA MCPHERSON (CONVOLVULACEAE)
}

\author{
Guillermina Murguia Sanchez \\ Judith MARQuez GuZMan \\ Guillermo Laguna Hernandez
}

Y

Margarita Ponce Salazar

Facultad de Ciencias

Universidad Nacional Autónoma de México

Apdo. Postal 70-356

04510 México, D.F.

\section{RESUMEN}

Ipomoea teotitlanica McPherson fue descrita a partir de un espécimen procedente de material de herbario del que se desconocían el fruto y la semilla.

En este trabajo se describe el ambiente de la localidad tipo en la que se establece una población de Ipomoea teotitlanica, así como las características estructurales de flor, fruto y semilla.

La morfología floral y la estructura de su fruto y semilla corresponden a la descrita para las especies integrantes de la serie Arborescentes: sépalos coriáceos, fruto dehiscente y semillas con largos tricomas marginales.

\begin{abstract}
Ipomoea teotitlanica McPherson was described from an unique herbarium specimen without fruits and seeds.

The environmental conditions of the type locality where Ipomoea teotitlanica is established and the structural features of its flowers, fruits and seeds are described here.

The morphology of fruit and seed corresponds to that described for the Arborescentes group: coriaceous sepals, dehiscent fruit and seed with long marginal trichomes.

\section{INTRODUCCION}

El género Ipomoea (Convolvulaceae) está formado por alrededor de 500 especies que presentan una distribución pantropical, con algunos taxa en latitudes templadas (McDonald, 1982). No sólo es el más grande sino también el más diverso de la familia en cuanto a formas de crecimiento y de adaptación se refiere (Pedraza, 1983). En México el género está representado por 140 a 150 especies (Austin y Pedraza, 1983; McDonald, 1991).
\end{abstract}


Dentro del género Ipomoea se encuentra un conjunto de especies de hábito arbóreo, arbustivo o de enredaderas leñosas que se ha diversificado en México (McDonald, 1992) y constituye la serie Arborescentes, sección Eriospermum, del subgénero Eriospermum (Austin, 1979; McDonald, 1991). Dado que las características del fruto y la semilla son parámetros importantes en la delimitación de la serie Arborescentes, nos hemos abocado al estudio morfológico y anatómico de estas estructuras.

Ipomoea teotitlanica pertenece a la serie Arborescentes (McDonald, 1991) y fue descrita por McPherson (1981) a partir de un solo espécimen (Gentry 22475 (GH)), procedente del Valle de Tehuacán-Cuicatlán (Oaxaca, México), el cual no incluía frutos ni semillas. En el presente trabajo se describen los resultados del estudio morfológico y anatómico de la flor, fruto y semilla de Ipomoea teotitlanica McPherson.

\section{METODOLOGIA}

Se realizaron tres recorridos (noviembre de 1989, febrero de 1990 y enero de 1991) en un transecto que abarcó $12 \mathrm{~km}$ al suroeste de San Antonio Nanahuatipan, Municipio de Teotitlán de Flores Magón, Oaxaca (entre los $18^{\circ} 7.8^{\prime}$ latitud norte, $97^{\circ} 4.6^{\prime}$ longitud oeste y los $18^{\circ} 3.4^{\prime}$ latitud norte, $97^{\circ} 12^{\prime}$ longitud oeste) en el Valle de Tehuacán-Cuicatlán. Se tomaron datos de distribución altitudinal (m s.n.m.) y del tipo de vegetación donde crece Ipomoea teotitlanica McPherson.

Se colectó material biológico para ejemplares de herbario, así como para estudios estructurales, que incluía: botones florales, flores, frutos y semillas, los cuales se procesaron en el mismo sitio de colecta para su posterior tratamiento, según la técnica de Johansen (1940).

Se estudió la anatomía de las semillas maduras con énfasis en la región micropilar, zona en que se distingue al cojincillo como estructura característica entre las especies de la familia Convolvulaceae, particularmente en la epidermis de la región adyacente al hilo y en la capa de esclerénquima de la cubierta seminal (Murguía, 1986).

Los ejemplares de herbario se depositaron en FCME, MEXU, ENCB, CHAPA, UAMIZ, IEB, XAL, UAS, UAG y CIFO.

\section{RESULTADOS}

Las pequeñas poblaciones de Ipomoea teotitlanica se distribuyen desde el cerro Tehuacanero en la orilla oriental del río Calapa, Municipio de Teotitlán de Flores Magón, hasta los alrededores de la localidad conocida como El Tambor, Municipio de Tepelmeme, Oaxaca (Fig. 1).

Ecológicamente I. teotitlanica se encuentra en altitudes que van de 800 a $1250 \mathrm{~m}$ s.n.m. y se establece en comunidades propias de la selva baja con dominancia de varias especies del género Bursera y de otros elementos arbóreos como Ceiba parvifolia, Cercidium spp., Eysenhardtia polystachya, Gyrocarpus mocinnoi, Cyrtocarpa procera e Ipomoea pauciflora; además se observan especies de Agave y Opuntia, Yucca, Neobuxbaumia tetetzo, Cephalocereus senilis, Beaucarnea gracilis, Lemaireocereus y Mammillaria. 
Murguía et al.: Estudio de Frutos y Semillas de Ipomoea teotitlanica

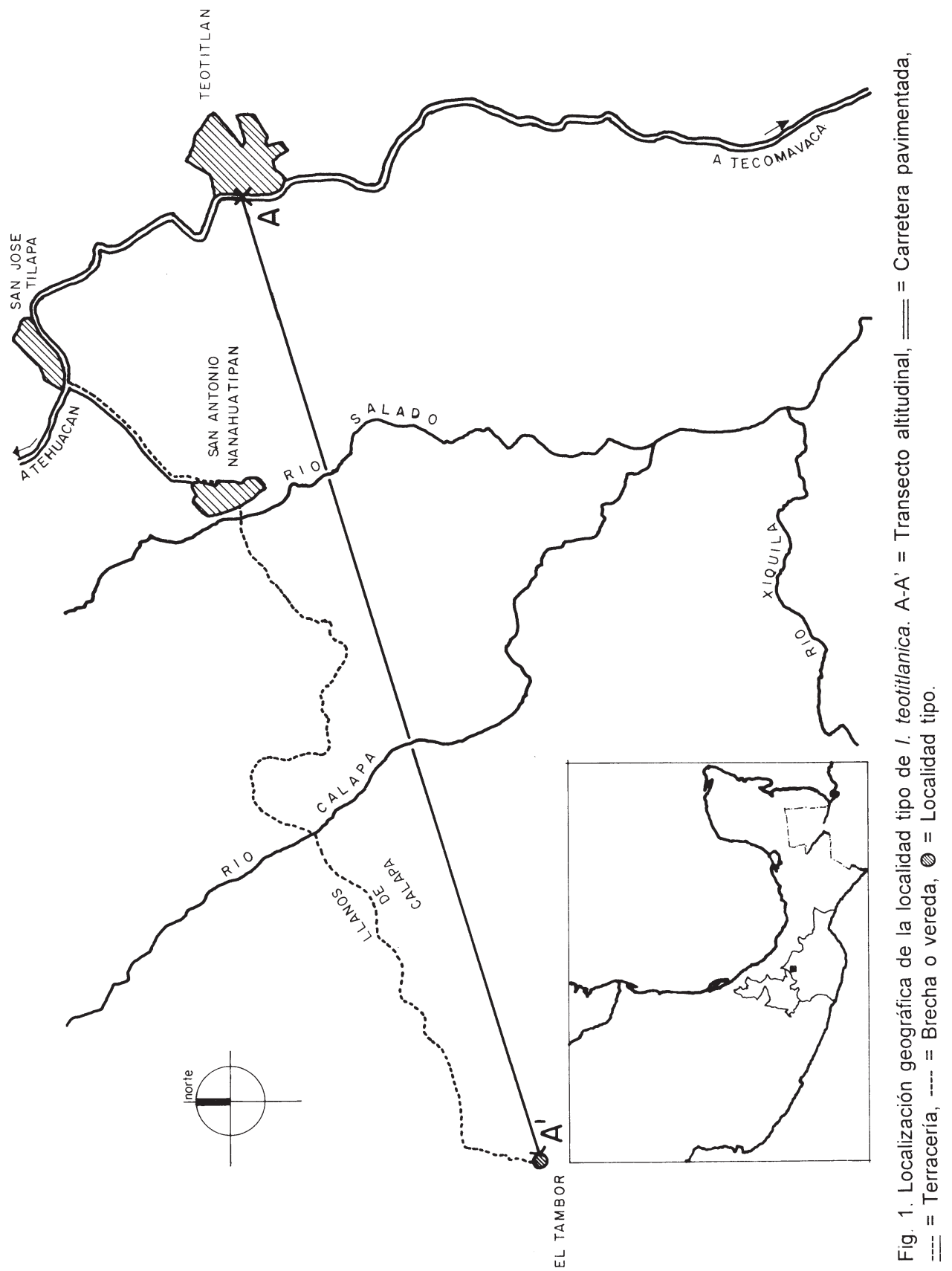


El clima de la región es del tipo seco árido y la precipitación es de alrededor de los $400 \mathrm{~mm}$ anuales (Dra. Irma Trejo, com. pers.).

El substrato geológico está formado por rocas sedimentarias del Cretácico Superior y los suelos son semidesérticos (sierozem) con acumulaciones de carbonato de calcio y humus, de escasos centímetros de espesor o inexistentes (Fuentes, 1972); son suelos pedregoso-arenosos en su textura superficial.

\section{Morfología vegetativa}

Hacia las partes bajas del transecto (800 m s.n.m., en el lado oriental del río Calapa) los manchones de $I$. teotitlanica son más bien ralos, estando mejor representados en la barranca de El Tambor, de modo que, en las inmediaciones de la localidad tipo (a partir de los 1000 m s.n.m.), la población es más numerosa.

La talla de los árboles de I. teotitlanica no es homogénea en el transecto. Esto puede deberse a la presencia de estadíos juveniles, maduros y viejos en la población o también a daño mecánico durante el desarrollo, pues hay individuos que se establecen a la orilla de veredas 0 al paso de animales y aun al borde de lechos de corrientes intermitentes, donde las violentas avenidas en la época de lluvias arrastran grandes cantidades de materiales rocosos.

Los árboles de Ipomoea teotitlanica miden entre 2 y $6 \mathrm{~m}$ de alto, presentan troncos grises de 5 a $30 \mathrm{~cm}$ de diámetro y la ramificación surge a partir de 20 a $80 \mathrm{~cm}$ del suelo (Fig. 2). Las ramas jóvenes son tomentosas y las maduras glabras. Los individuos producen látex blanco, que es liberado al corte de tallos, ramas, hojas, flores y frutos; se oxida al contacto con el aire tomando el color y la consistencia de una resina ambarina.

Las ramificaciones principales del sistema radicular se extienden paralelas u oblicuas a la superficie del substrato más que penetrar verticalmente.

Las hojas presentan haz pubescente de color verde obscuro y envés densamente tomentoso de color blanquecino, con 7 a 9 nervaduras principales a cada lado de la vena central.

Las hojas maduras miden de 2.5 a $4 \mathrm{~cm}$ de largo y de 3.2 a $5 \mathrm{~cm}$ de ancho, llevan pecíolos tomentosos de 1.2 a $2 \mathrm{~cm}$ de largo. El follaje conserva su color y textura naturales durante la preparación y montaje de los ejemplares de herbario (Fig. 3).

\section{Morfología floral}

Ipomoea teotitlanica florece en octubre y noviembre, aunque para enero aún se encuentran individuos produciendo flores. Estas crecen solitarias sobre pedúnculos de menos de $5 \mathrm{~mm}$ o ausentes y pedicelos de 0.8 a $1.5 \mathrm{~cm}$. Sépalos de 18 por $10 \mathrm{~mm}$, los más externos tomentosos, pudiendo ser glabros al momento de la dehiscencia del fruto. Corolas de 6.4 a $7.1 \mathrm{~cm}$ de largo y 5 a $9.3 \mathrm{~cm}$ de diámetro en el extremo superior, glabras y de color 3A6, amarillo claro brillante (Kornerup y Wanscher, 1978) (Fig. 4). Androceo de 2.6 a $4.8 \mathrm{~cm}$ de largo con anteras de $9 \mathrm{~mm}$ por $1.5 \mathrm{~mm}$ de ancho con pelos nectaríferos en la base de cada filamento. Gineceo de 3.7 a $4.6 \mathrm{~cm}$ de largo, con estigma bilobulado de $2 \times 3 \mathrm{~mm}$ (Fig. 4). 
Murguía et al.: Estudio de Frutos y Semillas de Ipomoea teotitlanica

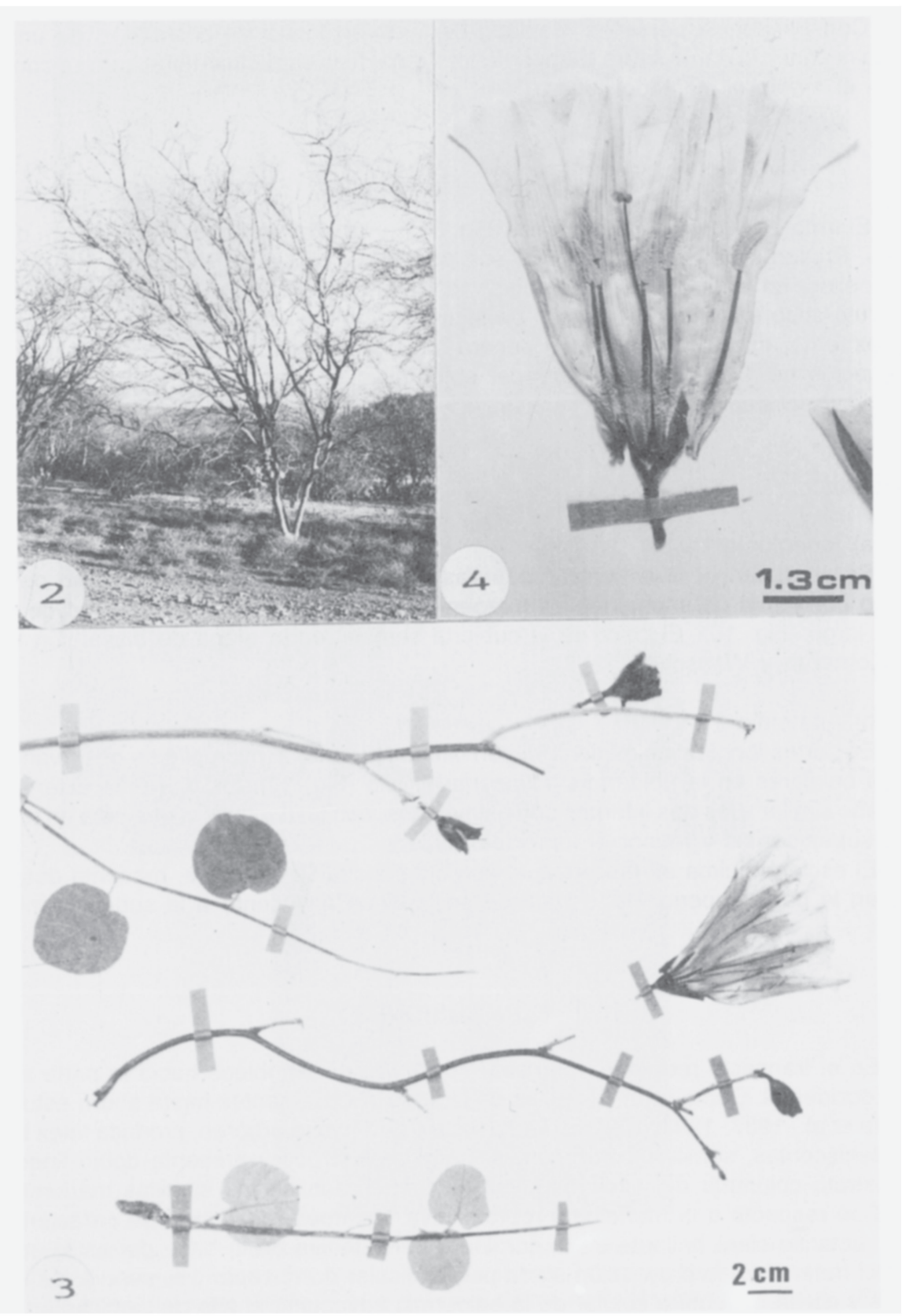

Fig. 2. Hábito. Fig. 3. Ejemplar del herbario. Fig. 4. Androceo y gineceo. 
Con frecuencia persisten residuos de látex en forma de cristales o de una frágil película micácea brillante sobre la superficie externa de la estructura floral, que se conservan aún en el ejemplar montado.

Fruto

El fruto es bilocular y dehiscente (Fig. 5), con cuatro valvas de 14 a $18 \mathrm{~mm}$ de largo (Fig. 6). Presenta dos estructuras que son poco usuales en las especies de este grupo: una de ellas, en el ápice de los carpelos, corresponde a la base del estilo que persiste en el fruto seco e impide el depósito de pigmento en esa zona (Figs. 7 y 8); la segunda, en el extremo apical del septo que separa los dos lóculos en el fruto, consiste de una prolongación de 1.5 a $2 \mathrm{~mm}$, al parecer epidérmica, a cada lado y perpendicular al eje longitudinal del septo (Fig. 9).

Semillas maduras

a) Morfología

Se producen generalmente 4 semillas por fruto, con medidas promedio de $8 \mathrm{~mm}$ de largo por $4 \mathrm{~mm}$ de ancho, en los márgenes laterales provistas de tricomas de 9 a 11 $\mathrm{mm}$ de largo (Fig. 10). El color de la cubierta seminal en la etapa de dispersión es $6 \mathrm{F5}$ café (Kornerup y Wanscher, 1978).

\section{b) Anatomía}

En cortes longitudinales de la región hilar adyacente al micrópilo se observan cuatro estratos celulares en la epidermis adyacente al hilo (Fig. 11): los dos más externos son de tamaño similar y los dos internos son asimétricos, con talla mayor el que está en contacto con la subepidermis y menor el inmediato superior.

El esclerénquima en empalizada, con 3 a 5 estratos celulares, presenta dos líneas claras en la parte superior del estrato de esclereidas adyacente a la subepidermis (Fig. 12).

\section{CONCLUSIONES}

En el transecto recorrido, Ipomoea teotitlanica se establece hacia la parte alta, en el lado occidental. Como otras especies de la serie Arborescentes hasta ahora estudiadas (Murguía et al., 1987, 1993, 1994), I. teotitlanica es de hábito arbóreo, produce látex blanco, frutos dehiscentes, semillas con tricomas marginales largos y presenta doble línea clara en el estrato columnar del esclerénquima en empalizada de las semillas maduras.

Con respecto a la morfología reproductiva, Ipomoea teotitlanica se caracteriza por el color amarillo claro brillante de la corola, la persistencia de la base del estilo sobre el ápice del fruto seco, la proyección alada perpendicular en el septo que separa los lóculos del fruto y por la estructura celular de la epidermis adyacente al hilo de semillas maduras, que consiste de cuatro estratos celulares: dos externos simétricos y dos internos asimétricos. 

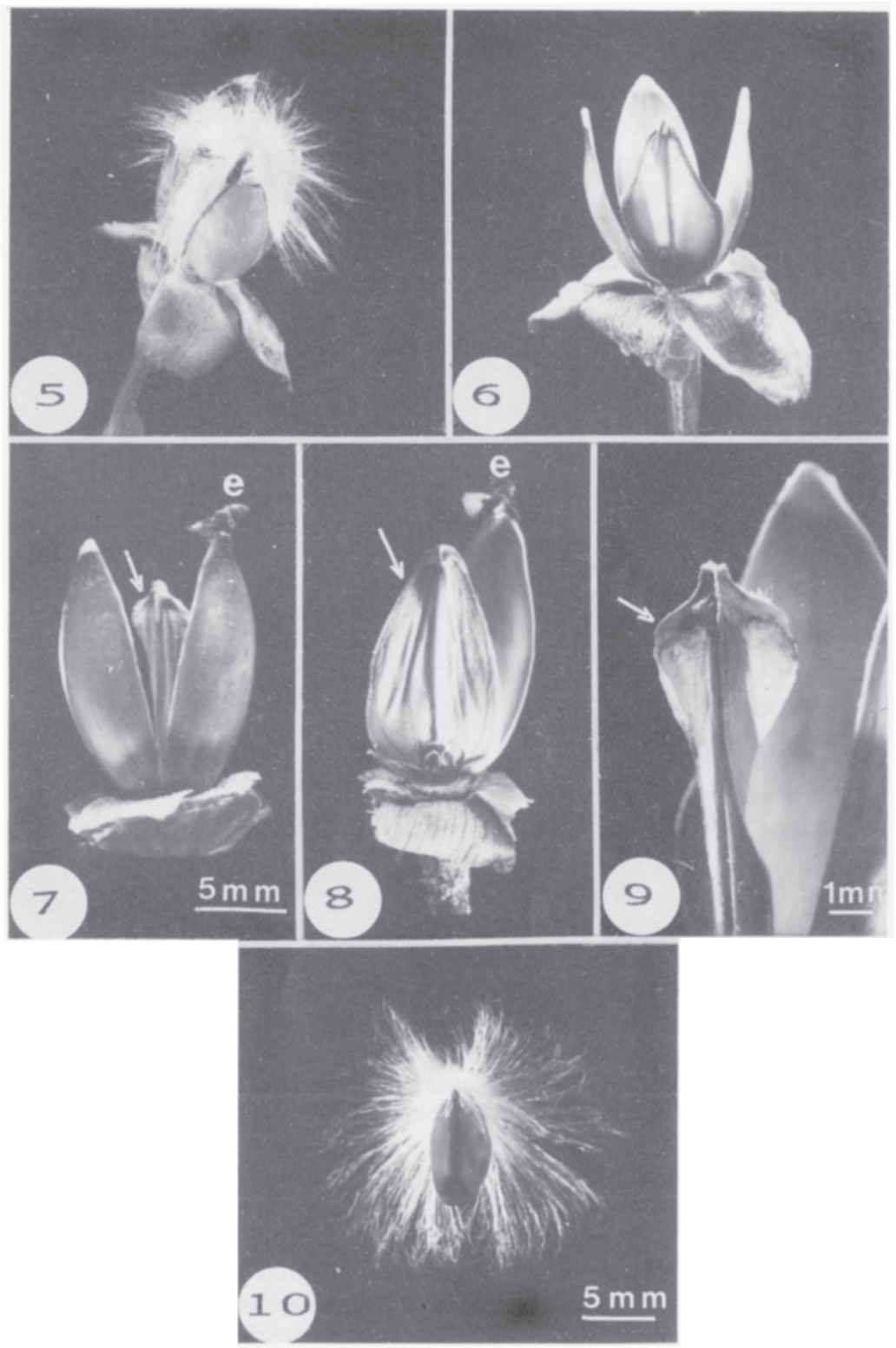

Fig. 5. Fruto dehiscente. Fig. 6. Se muestran tres de sus cuatro valvas y el septo que separa los dos lóculos. Fig. 7. Base del estilo (e) sobre una de las valvas del fruto y vista lateral del septo (flecha). Fig. 8. Vista frontal del septo (flecha) y base del estilo sobre una de las valvas (e). Fig. 9. Vista lateral del septo (flecha). Obsérvese la proyección alada perpendicular al eje longitudinal. Fig. 10. Semilla madura con tricomas en los márgenes laterales. Vista ventral. 


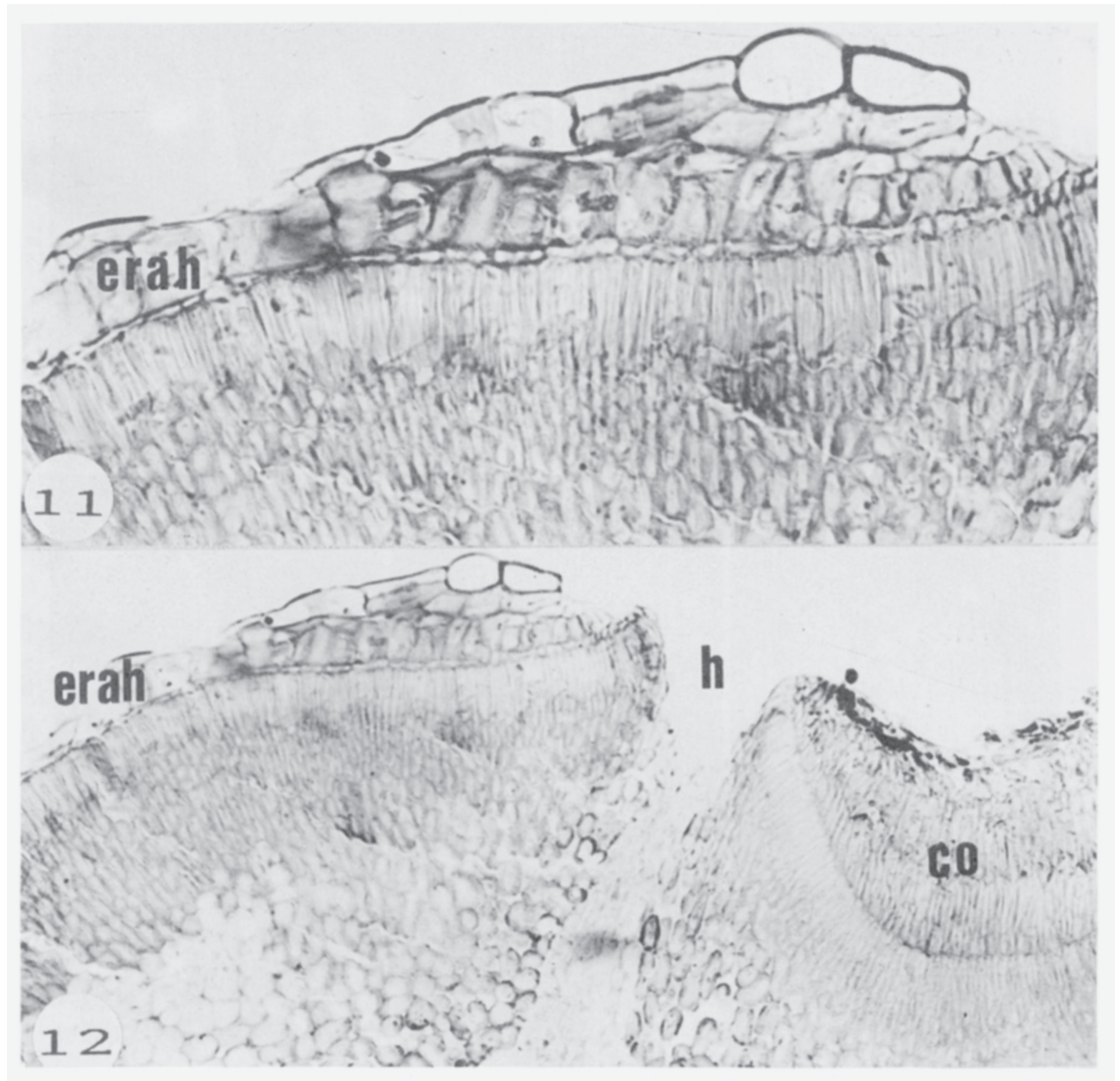

Fig. 11. Epidermis de la región hilar adyacente al micrópilo (erah). Se observan dos estratos celulares externos simétricos y dos estratos celulares internos asimétricos. Fotomicrografía 346x. Fig. 12. Corte mediano de la cubierta seminal en la región micropilar. Se observa el cojincillo (co), hilo (h) y epidermis de la región hilar adyacente al micrópilo (erah). Fotomicrografía 160x.

\section{AGRADECIMIENTOS}

Agradecemos la valiosa colaboración de la familia Arriaga, de San Antonio Nanahuatipan, Oaxaca, para la realización del trabajo de campo en esta investigación; al Biól. Ricardo Wong G. su entusiasta participación en las colectas y en el trabajo de fotografía en el campo; al Biól. Antonio Hernández, del Laboratorio de Microcine de la Facultad de Ciencias, la preparación del material que ilustra el presente trabajo. A las siguientes personas, la revisión y sugerencias al contenido del manuscrito: Dr. Fernando Chiang C., Dra. Alicia Brehú F., Dra. R. Irma Trejo V., Biól. Pedro Tenorio L., y Biól. M. Angel Soto A. 


\section{LITERATURA CITADA}

Austin, D. F. 1979. An infrageneric classification for Ipomoea (Convolvulaceae). Taxon 28(4): 359-361. Austin, D. F. y R. A. Pedraza. 1983. Los géneros de convolvuláceas en México. Bol. Soc. Bot. Méx. (4): 3-16.

Fuentes A., L. 1972. Regiones naturales del estado de Puebla. Instituto de Geografía. Universidad Nacional Autónoma de México. México, D.F. 143 pp.

Johansen, D. A. 1940. Plant microtechnique. McGraw-Hill Book Co. Inc. Londres. págs. 107, 126-154.

Kornerup, A. y J. H. Wanscher. 1978. Methuen handbook of colour. Eyre Methuen. Londres. 252 pp.

McDonald, A. J. 1982. Biosystematics of the Ipomoea tricolor complex (Convolvulaceae). Ph. D. Dissertation. The Univ. of Texas at Austin. Austin, Texas.

McDonald, A. J. 1991. Origin and diversity of Mexican Convolvulaceae, Anales Inst. Biol. Univ. Nac. Autón. México. Ser. Bot. 62(1): 65-82.

McDonald, A. J. 1992. Evolutionary implications of typical and anomalous secondary growth in arborescent Ipomoea (Convolvulaceae). Bull. Torrey Bot. Club 119: 262-267.

McPherson, G. 1981. Studies in Ipomoea (Convolvulaceae): I. The Arborescens group. Ann. Missouri Bot. Gard. 68: 527-545.

Murguía S., G. 1986. Estudio comparativo de semillas maduras de dos especies arbóreas del género Ipomoea (Convolvulaceae). Tesis profesional. Facultad de Ciencias. Universidad Nacional Autónoma de México, México. 60 pp.

Murguía S., G., R. M. Ponce S. y J. Márquez G. 1987. Estudio comparativo de dos especies arbóreas del género Ipomoea (Convolvulaceae). Resúmenes del X Congreso Mexicano de Botánica. Guadalajara, Jalisco. trabajo 572.

Murguía S., G., J. Márquez-Guzmán y A. Martínez M. 1993. Morfología floral en la serie Arborescentes (Ipomoea, Convolvulaceae). Resúmenes del XII Congreso Mexicano de Botánica. Mérida, Yucatán. p.58.

Murguía S., G., J. Márquez-Guzmán y A. Martínez M. 1994. Anatomía de semillas en la serie Arborescentes (Ipomoea, Convolvulaceae). Resúmenes del VI Congreso Latinoamericano de Botánica. Mar del Plata, Argentina. p.386.

Pedraza, R. A. 1983. Estudio palinológico de la familia Convolvulaceae en México. I. Géneros Ipomoea L. y Turbina Raf. Biótica 8(4): 387-411. 